\title{
THE SEPARATION THEOREM FOR QUASI-CLOSED SETS
}

\author{
JOHN H. V. HUNT AND ADALBERTO GARCÍA-MÁYNEZ
}

In the memory of Dr. Gordon T. Whyburn, our professor and adviser

ABSTRACT. The concepts of "closed set, separation and $n$-cell" are generalized to "quasi-closed set, weak separation and locally cohesive space," respectively. It is then proved that any quasiclosed set $L$, which weakly separates two closed subsets $A, B$ in a locally cohesive $T_{1}$-space $X$, contains a closed set $K$ which separates $A-K$ and $B-K$ in $X$.

1. Introduction. In this article we complete a sequence of arguments concerning quasi-closed sets that appear in [3].

In [3] Whyburn proves the following theorem.

Theorem. Let $A$ and $B$ be disjoint nondegenerate closed and connected sets in a locally cohesive $T_{1}$-space $X$. Any quasi-closed set $L$ which weakly separates $A$ and $B$ in $X$ contains a closed set $K$ which separates $A-K$ and $B-K$ in $X$.

In the "Concluding Remarks" of [3] Whyburn shows that the requirement that $A$ and $B$ be nondegenerate can be deleted. He also mentions that the condition that $A$ and $B$ be connected can be replaced by the requirement that each of them be of dimension $>0$ at each point.

In this article we show that $A$ and $B$ can in fact be arbitrary closed sets. The theorem to this effect appears in $\$ 2$. We call it the separation theorem for quasi-closed sets.

We are grateful to Dr. Whyburn for pointing out, in Appendix I of [4], that the results in the "Concluding Remarks" of [3] are partial versions of our theorem in $\$ 2$.

2. We first define the necessary terms. We follow the definitions in [3], except for two changes. Firstly, our definition of "unicoherence between two subsets" is weaker than the definition in $\$ 5$ of [2], on which the definition of "local cohesiveness" in [3] is based. Secondly, we define "local cohesiveness" for arbitrary spaces. This means that

Received by the editors January 14, 1970.

AMS 1969 subject classifications. Primary 5423; Secondary 5455.

Key words and phrases. Quasi-closed set, canonical region, locally cohesive space. weak separation. 
we usually have to include certain separation properties in the statements of our results.

In all the definitions that follow $X$ is an arbitrary topological space unless otherwise stated.

A set $E$ in a space $X$ is quasi-closed in $X$ if each point in $X-E$ has a base of neighbourhoods whose frontiers do not meet $E$.

Let $E$ and $F$ be two disjoint subsets of a connected space $X$. We say that $X$ is unicoherent between $E$ and $F$ if however $X$ is expressed as the union of two connected closed sets $M$ and $N$ such that $M-N$ and $N-M$ contain $E$ and $F$, respectively, $M \cap N$ is always connected. If $p$ is a point of a space $X$, we say that $R$ is a canonical region about $p$ in $X$ if $R$ is a connected neighbourhood of $p$, the frontier $\operatorname{Fr} R$ of $R$ is connected, and $\bar{R}$ is unicoherent between $\{p\}$ and $\operatorname{Fr} R$ (or equivalently, in case $X$ is connected, $X$ is unicoherent between $\{p\}$ and $X-R)$. A space $X$ is locally cohesive if each of its points has a base of canonical regions. Notice that a locally cohesive space is locally connected.

Let $E, F$ and $L$ be subsets of a space $X$. We say that $L$ separates $E$ and $F$ in $X$ if $X-L$ is the union of two sets $M$ and $N$ which contain $E$ and $F$, respectively, and which are separated in $X$ ( $M$ and $N$ are separated in $X$ if $\bar{M} \cap N=\varnothing=M \cap \bar{N})$. We say that $L$ weakly separates $E$ and $F$ in $X$ if no component of $X-L$ meets both $E$ and $F$. Notice that we may have $E \cap F \cap L \neq \varnothing$ in this last definition.

Before giving the theorem, we state two simple lemmas. These can be found as statements in $\$ 1$ of [3]. They are, in any event, easily proved on the basis of our definitions.

2.1 Lemma. If $R$ is a canonical region about a point $p$ in a locally cohesive space $X$, and $K$ is a closed set in $\bar{R}$ that separates $p$ and $\mathrm{Fr} R$ in $\bar{R}$, then there is a canonical region $S$ about $p$ such that $\bar{S} \subset R$ and Fr $S \subset K$.

2.2 Lemma. If $L$ is a quasi-closed set in a locally cohesive regular space $X$, then each point of $X-L$ has a base of canonical regions whose frontiers do not meet $L$.

2.3 Theorem. Let $A$ and $B$ be closed sets in a locally cohesive regular $T_{1}$-space $X$. Any quasi-closed set $L$ which weakly separates $A$ and $B$ in $X$ contains a closed set $K$ which separates $A-K$ and $B-K$ in $X$.

Proof. We notice that we may suppose without loss of generality that $X$ is connected, for on the one hand the restriction of $L$ to a component of $X$ is quasi-closed, and on the other hand the union of a 
collection of closed sets, each contained in a component of $X$, is closed. Thus we shall suppose that $X$ is connected.

We first consider the case of a point $p \in A-L$ which lies in a nondegenerate component $H_{p}$ of $X-L$, and we show that there is a region $G_{p}$ about $p$ which does not meet $B$ and for which $\operatorname{Fr} G_{p} \subset \bar{H}_{p}$ $\cap L$.

First notice that $\bar{H}_{p}-H_{p} \subset L$; for if $x \in \bar{H}_{p}-L$, then $H_{p} \cup\{x\}$ is a connected set in $X-L$ and so is contained in $H_{p}$. Now let $V$ be the union of all the components of $X-\bar{H}_{p}$ that meet $B$. Then $\operatorname{Fr} V \cap H_{p}$ $=\varnothing$. For let $x \in H_{p}$ and let $R$ be a canonical region about $x$ which neither meets $B$ nor contains $H_{p}\left(H_{p}\right.$ is nondegenerate and $X$ is a $T_{1^{-}}$ space) and whose boundary $\operatorname{Fr} R$ does not meet $L$. Then $H_{p}$ meets both $R$ and its complement and so contains $\operatorname{Fr} R$. However, each component of $V$ meets $X-R$ but not $\mathrm{Fr} R$, and so does not meet $R$. Consequently $V$ does not meet $R$ and $x \notin \operatorname{Fr} V$. Thus $\operatorname{Fr} V \subset \bar{H}_{p}-H_{p}$. Thus $X-\left(\bar{V} \cup\left(B \cap \bar{H}_{p}\right)\right)$ is a neighbourhood of $p$ which does not meet $B$ and whose frontier is contained in $\bar{H}_{p} \cap L$. If we let $G_{p}$ be the component of $X-\left(\bar{V} \cup\left(B \cap \bar{H}_{p}\right)\right)$ that contains $p$, then $G_{p}$ is a region about $p$ which does not meet $B$ and for which $\operatorname{Fr} G_{p} \subset \bar{H}_{p} \cap L$.

Now we consider the case of a point $p \in A-L$ which lies in a degenerate component of $X-L$, and we show that there is a region $G_{p}$ about $p$ whose closure does not meet $B$ and whose boundary is a connected subset of $L$.

Let $R$ be a canonical region about $p$ whose complement is nondegenerate and contains $B$ and such that $\operatorname{Fr} R \cap L=\varnothing$. Then $L \cap R$ is a quasi-closed set, and we assert that it weakly separates the closed sets $\{p\}$ and $X-R$. For let $H$ be the component of $X-L \cap R$ that contains the connected closed set $X-R$. Then $H \cap \bar{R}$ is connected, because it is a closed subset of $H$ which contains the connected set Fr $R$. It follows that $p$ cannot belong to $H$, because if it did $\{p\}$ $\cup(H \cap \bar{R})$ would be a nondegenerate connected subset of $X-L$, contradicting the assumption that $p$ lies in a degenerate component of $X-L$. Since $H$ is nondegenerate, there is by the second paragraph of this proof a region $G$ which contains $H$ and does not meet the closed set $\{p\}$, and whose boundary lies in $L \cap R$. Let $G_{p}$ be the component of $X-\bar{G}$ that contains $p$. Then $\operatorname{Fr} G_{p}$ is connected. For $X=\bar{G}_{p}$ $\cup\left(X-G_{p}\right)$, where $\bar{G}_{p}$ and $X-G_{p}$ are two connected closed subsets of $X$ such that $\{p\} \cap\left(X-G_{p}\right)=\varnothing$ and $(X-R) \cap \bar{G}_{p}=\varnothing$. Therefore, since $R$ is a canonical region about $p$, Fr $G_{p}=\bar{G}_{p} \cap\left(X-G_{p}\right) \cap \bar{R}$ is connected. That is, $G_{p}$ is a region about $p$ whose closure does not meet $B$ and whose boundary is a connected subset of $L$. 
We shall suppose hereafter that $B$ is nondegenerate for if $B$ is degenerate we can prove the theorem by interchanging the letters " $A$ " and " $B$ " in the second and fourth paragraphs of the proof when $B C X-L$, and by removing the set $B$ from $X$ when $B C L$.

Now we show that $\operatorname{Fr} \cup G_{p} \subset L$, the union being taken over all points $p \in A-L$. Suppose that $x \in\left(\operatorname{Fr} \cup G_{p}\right)-L$. Then $x \notin A$, so there is a canonical region $R$ about $x$ such that $R \cap A=\varnothing, R D B$ and Fr $R \cap L=\varnothing$. Then $G_{p}$ meets $R$ for some $p \in A-L$, and consequently Fr $G_{p}$ meets $R$, because $R$ is connected and not contained in $G_{p}$. It follows that $p$ cannot lie in a degenerate component of $X-L$, for in this case $\operatorname{Fr} G_{p}$ is a connected subset of $L$ and so is contained in $R$. Thus, since $X-R$ is a connected set in the complement of $\operatorname{Fr} G_{p}$ which meets $B, \operatorname{Fr} G_{p}$ separates not only $p$ and $B$ but also $p$ and $X-R$. That is, $p \in R$, which is false because $R \cap A=\varnothing$. So $p$ lies in a nondegenerate component $H_{p}$ of $X-L$. Further, since Fr $G_{p} \subset \bar{H}_{p}, H_{p}$ meets both $R$ and its complement, and so meets and contains $\operatorname{Fr} R$. However $x \notin \bar{G}_{p}$, so there is a point $q \in A-L$ such that $G_{q}$ meets $R-\bar{G}_{p}$, and, as before, $q$ lies in a nondegenerate component $H_{q}$ of $X-L$ which contains Fr $R$. But this implies that $H_{p} \cap H_{q} \neq \varnothing$, and so $H_{p}=H_{q}$. Consequently $G_{p}=G_{q}$, by construction, which is a contradiction.

Let $K=\left(\operatorname{Fr} \cup G_{p}\right) \cup\left(A-\cup G_{p}\right)$, the union again being taken over all $p \in A-L$. Then $K$ is a subset of $L$ which is closed in $X$, and it separates $A-K$ and $B-K$ in $X$.

3. In conclusion we wish to point out the relation between certain results in [1] and [4] and Theorem 2.3 above.

In Appendix I of [4] the proof of (2.3) is broken into three steps:

(A) Theorem 1, p. 58;

(B) Theorem 2, p. 59;

(C) Separation theorem, p. 61.

It is shown in [4] that (A) and (B) imply (C), which is our Theorem 2.3. It will be noticed that it also follows immediately that (C) implies (A) and (B).

We shall use the following notation in discussing the results of [1]. We shall denote by $X$ a locally cohesive regular $T_{1}$-space; by $L$ a quasi-closed set in $X$; by $G$ the set $X-L$; by $\&$ the decomposition of $G$ in to its components and by $p$ the natural projection from $G$ onto the decomposition space $G / \mathrm{g}$. For each subset $A$ of $X$, we shall denote by $H_{A}$ the union of components of $G$ which meet $A$, and finally, for each subset $C$ of $G, C^{\sim}$ will stand for the closure of $C$ in the subspace $G$; that is, $C^{\sim}=\bar{C} \cap G$. 
Observe that two subsets $A, B$ of $X$ are weakly separated by $L$ if and only if $H_{A} \cap H_{A}=\varnothing$.

The following results are proved in [1]:

(D) If $A$ and $B$ are subsets of $X$, then there is a closed set $K \subset L$ separating $A-L$ and $B-L$ if and only if $\bar{H}_{A}$ and $\bar{H}_{B}$ are weakly separated by $L$.

(R) For each closed subset $A$ of $X, H_{A}=\bar{H}_{A} \cap G$.

Proof of (R). Assume $x \in G \cap\left(\bar{H}_{A}-H_{A}\right)$. Then $x \notin A$ and thus there is a canonical region $U$ about $x$ such that $\operatorname{Fr} U \subset G$ and $U \cap A$ $=\varnothing$. Hence $U \cap A_{\alpha} \neq \varnothing$, where $A_{\alpha}$ is a component of $G$ in $H_{A}$. Notice $(X-U) \cap A_{\alpha} \neq \varnothing$, for $A_{\alpha} \cap A \neq \varnothing$ and $U \cap A=\varnothing$. Therefore $A_{\alpha}$ meets and contains $\operatorname{Fr} U$. Observe that $x \notin \bar{A}_{\alpha}$ so that there is a canonical region $U^{\prime}$ about $x$ such that $U^{\prime} \subset U$ and $U^{\prime} \cap A_{\alpha}=\varnothing$. Since $x \in \bar{H}_{A}$, $U^{\prime} \cap A_{\alpha^{\prime}} \neq \varnothing$ for some component $A_{\alpha^{\prime}}$ of $G$ in $H_{A}$. Evidently $U \cap A_{\alpha^{\prime}}$ $\neq \Phi$, so as before we get Fr $U \subset A_{\alpha^{\prime}}$, a contradiction.

We can now prove the following two theorems with the help of our Theorem 2.3 and remark (R). Theorem (D) is a consequence of 3.2 below.

3.1. The following three properties are equivalent $(A$ and $B$ are subsets of $X$ ):

(1) $\bar{A}$ and $\bar{B}$ are weakly separated by $L$.

(2) $H_{\bar{A}}$ and $H_{\bar{B}}$ are weakly separated by $L$.

(3) There exists a closed set $K \subset L$ separating $A-K$ and $B-K$. Also,

3.2. The following three properties are equivalent:

(4) $(A \cap G)^{-}$and $(B \cap G)^{-}$are weakly separated by $L$.

(5) $\bar{H}_{A}$ and $\bar{H}_{B}$ are weakly separated by $L$.

(6) There exists a closed set $K \subset L$ separating $A-L$ and $B-L$.

Proof of 3.1. The equivalence of (1) and (2) is obvious. The implication $(1) \Rightarrow(3)$ is a consequence of our Theorem 2.3 (and indeed, it is equivalent to 2.3 , as will be observed below). In order to show that $(3) \Rightarrow(1)$, we notice that (3) is equivalent to this proposition:

(3)' There exist disjoint open sets $V_{a}, V_{b}$ such that $A \cap V_{b}=B \cap V_{a}$ $=\varnothing$ and $G \subset V_{a} \cup V_{b}$.

Thus (3) can be replaced by:

(3)" There exists a closed set $K \subset L$ separating $\bar{A}-K$ and $\bar{B}-K$.

Now the implication $(3)^{\prime \prime} \Rightarrow(1)$ follows directly from the definition of separating and weak separating.

Proof of 3.2. To prove the equivalence of (4) and (5), we use (R). Indeed, (R) tells us that the decomposition $\mathcal{G}$ of $G$ is upper semicontinuous or, equivalently, that the mapping $p: G \Rightarrow G / \mathcal{G}$ is closed. 
Therefore

$$
\begin{aligned}
H_{\bar{H}_{A}} & =p^{-1}\left[p\left(H_{A}\right)\right]=p^{-1}\left[p\left(H_{A}\right)^{-}\right]=p^{-1}\left[p(A \cap G)^{-}\right] \\
& =p^{-1} p\left[(A \cap G)^{\sim}\right]=H_{(A \cap G)^{-}} .
\end{aligned}
$$

Similarly, $H_{\bar{H}_{B}}=H_{(B \cap G)-}$, so that $(4) \Leftrightarrow(5)$.

To prove $(4) \Rightarrow(6)$, we obtain from 3.1 a closed set $K \subset L$ separating $(A \cap G)^{-}-K$ and $(B \cap G)^{--} K$, and since these sets contain $A \cap G$ and $B \cap G$ respectively, $K$ separates $A \cap G$ and $B \cap G$. Finally, the implication $(6) \Rightarrow(4)$ is proved in the same way as $(3) \Rightarrow(1)$.

\section{REFERENCES}

1. A. García-Máynez, Ph.D. Thesis, University of Virginia, Charlottesville, Va., 1968.

2. G. T. Whyburn, Loosely closed sets and partially continuous functions, Michigan Math. J. 14 (1967), 193-205. MR 34 \#8387.

3. — Quasi-closed sets and fixed points, Proc. Nat. Acad. Sci. U.S.A. 57 (1967), 201-205. MR 35 \#1006.

4. G. T. Whyburn, assisted by J. H. V. Hunt, Notes on functions and multifunctions, University of Virginia, Charlottesville, Va., 1966/67 (mimeographed notes).

University of Virginia, Charlottesville, Virginia 22901 